\title{
Necesidad de adquirir competencias en valores y actitudes
}

\author{
José Luis Villanueva Marcos
}

Agencia Laín Entralgo

La educación médica en sus distintos niveles (pregrado, formación de especialistas y formación continuada) debe garantizar la formación de profesionales bien cualificados, que respondan a las necesidades del sistema sanitario. Para conseguirlo es necesario que se definan las competencias que a través de la formación se desean alcanzar. Dichas competencias se adquieren mediante un proceso dinámico y longitudinal en el tiempo y posibilitan el desarrollo eficaz de la profesión médica en todas las situaciones prácticas que se planteen. Por este motivo es muy importante que el médico recién licenciado haya adquirido en las facultades de medicina una serie de conocimientos, habilidades y actitudes que tendrá que continuar desarrollando durante los años de residencia y después durante toda su vida en el ejercicio profesional; para ello, es necesario que los Sistemas de Salud y las Universidades vayan de la mano en la formación de los estudiantes de Medicina. Tanto las instituciones sanitarias como educativas están de acuerdo en que además de todo aquello que podríamos englobar dentro de la "pericia clínica", es preciso que la formación abarque también "los valores humanos".
En los últimos años se han producido una serie de cambios que han hecho mejorar la percepción que los pacientes tienen de la Sanidad de nuestro país, y junto con ellos coexisten realidades que nunca van a cambiar, como es la naturaleza biológica del hombre. La naturaleza biológica de todo hombre es intangible, en el sentido de que es constitutiva de la identidad personal del individuo durante todo el curso de su historia. Cada persona humana, en su singularidad absolutamente única, debe ser tenida en cuenta. Sobre la base de esta visión antropológica se deben encontrar los criterios fundamentales para tomar decisiones en el trato con los pacientes. El sujeto humano enfermo, capaz de inteligencia y libertad es respetable a pesar de sus limitaciones y por ello si se le trata según criterios no basados en su realidad integral, se cae en el riesgo de atentar contra su dignidad o de exponerle al capricho ajeno, privándole de su autonomía. Todo esto está en la base de una serie de valores que siempre han necesitado los médicos para su ejercicio profesional a lo largo de la historia, desde Hipócrates hasta nuestros días y que se han reflejado en diferentes códigos y declaraciones (Tabla I)

TABLA I. Códigos y Declaraciones

- Juramento de Hipócrates (s V aC)

- Consejos de Esculapio a los que van a dedicarse a la medicina (Templo Esculapio s IV aC, Galeno S II dC)

- Plegaria del médico (s XII)

- Juramento de Maimónides (1135-1204)

- Código de Montpellier (Consejo de la federación de los Sindicatos Médicos de L'Herault 1924)

- Código de Nüremberg (Tribunal Internacional de Nürembreg 1946)

- Declaración Universal de los derechos Humanos (Asamblea General de las Naciones Unidas 1948)

- Código Internacional de Ética Médica (Asociación Médica Mundial III Asamblea general. Londres 1949. Revisiones de Sydney 1968 y Venecia 1983)

- Resolución 37/194 de la Asamblea General de las Naciones Unidas 1982

- Comité permanente de médicos europeos. Sobre la limitación de los recursos de salud y ética de los médicos. 1992

- Convenio Europeo sobre los Derechos Humanos y Biomedicina (Oviedo 1997)

- Carta de los derechos Fundamentales de la Unión Europea (2000)

- Declaración de la Asociación Médica Mundial sobre el trato a prisioneros y detenidos (Tokio 1975, Divonne-les-Bains 2006) 
No siempre se han seguido estas recomendaciones. Por ejemplo, en la época griega, en la que la ciencia médica estaba bien estructurada y requería largo tiempo de aprendizaje, existía otro tipo de práctica empírica, que no conocía el porqué ni el cómo de sus actos y que constituía una amenaza para los profesionales y los pacientes. Por otra parte, la organización misma de la sociedad griega atentaba contra lo que hoy llamaríamos profesionalismo: El ejercicio de la medicina era discriminatorio, distinto según el estrato social del enfermo. El siguiente texto de Platón refleja muy bien este aspecto.

“..... A los esclavos los tratan por lo general los esclavos, ya sea corriendo de un lado a otro o permaneciendo en sus consultorios; ninguno de tales médicos da ni admite la menor explicación sobre la enfermedad de cada uno de estos esclavos, sino que prescribe lo que la práctica rutinaria le sugiere, como si estuviere perfectamente al tanto de todo y con la arrogancia de un tirano, y pronto salta de alli en busca de otro esclavo enfermo, y así alivia a su dueño del cuidado de atender a tales pacientes"

(Platón. La leyes)

Otro ejemplo de la época contemporánea. Leo Alexander, en un magnífico artículo publicado en $\mathrm{N}$ Engl J Med el 14 de julio de 1949, recuerda que la ciencia bajo las dictaduras queda subordinada a la filosofía que las guía. Todos recordamos los horrores de la Alemania nazi, pero años antes de que los Nazis tomaran el poder en Alemania, ya se había lanzado una campaña de propaganda contra las actitudes tradicionales en la atención de los enfermos crónicos y a favor del utilitarismo. En este sentido, el citado autor menciona en su artículo como en 1931, durante una reunión de psiquiatras en Baviera se había debatido sobre la necesidad de esterilizar y/o eliminar a los pacientes con enfermedades mentales crónicas. Posteriormente vino lo que vino.

Actualmente podemos encontrarnos a algunas personas con desorientación respecto a los valores que se necesita tener en cuenta para moverse por la vida. En este sentido, es ilustrativa la respuesta a una de las preguntas de un reciente cuestionario del CIS en nuestro país en la que, un $41 \%$ de las personas entrevistadas, afirma que le cuesta discernir lo que está bien de lo que está mal. La realidad puede variar de un país a otro, pero esta desorientación está bastante generalizada y, unida a una notable falta de madurez, podría ser la causa de la situación que se describe en las escuelas de medicina de Minnesota (Clínica Mayo, campus de Minneapolis y campus de Duluth), en la que el $45 \%$ de los alumnos se encuentran bajo el efecto del Burnout. Está muy extendida de nuestra civilización occidental la orientación básica que considera, por un lado, como fin último de la vida humana el pasarlo bien, o al menos sentirse bien y, por otro, como uno de los deberes éticos más altos, aprovechar al máximo el mundo personal con el mayor incremento posible de sensaciones placenteras. En este contexto, parece que lo relevante no es lo que un hombre es, sino como está, en qué situación se encuentra y que tipo de sensaciones y vivencias experimenta. Se dice que el hombre no hace otra cosa que pasar por determinadas situaciones o estados.

De cara a los rumbos que toman nuestras decisiones, merece la pena recalcar la importancia que tiene el ajuste de nuestras nociones antropológicas (la verdad de nuestras concepciones teóricas acerca del mundo y de la vida) a la realidad. Para llegar a ser la clase de persona a la que de modo natural y habitual "le sale" hacer lo que hay que hacer, no hay que partir de una situación de excelencia intelectual o tener una dotación genética específica. Más bien se precisa un proceso educativo, largo y progresivo, que comienza en la infancia de cada uno, y se continúa a lo largo de la vida, desembocando en la Facultad de Medicina, en el periodo de residencia y como profesional senior más adelante. Es muy importante transmitir, sobre todo a los alumnos cuando comienzan su estancia en el hospital y a los médicos especialistas en formación, el valor del esfuerzo sostenido; en un contexto como el nuestro, en el que se sobrevalora el triunfo, el éxito -a veces episódico y pasajero- es especialmente urgente transmitir el mensaje que subraya la importancia de la tenacidad, de la constancia y del orden en el trabajo y en el estudio. La improvisación puede valer a veces, pero suele llegar más lejos, y más al fondo, quien es capaz de vencer su pereza de modo regular, quien ha construido hábitos intelectuales y de actuación que poco a poco van dando estabilidad a los proyectos personales y coherencia a la vida que lleva. Ésta persona sabe que lo que vale cuesta esfuerzo, y que no es posible adquirir una soltura en el manejo con los pacientes, atender con paciencia a un familiar en una guardia complicada, saber presentar bien una sesión clínica, enseñar a un residente de años inferiores, publicar en revistas inter- 
nacionales con buen factor de impacto, etc, si no es después de un largo entrenamiento. Y todo lo anterior haciéndolo compatible con una dedicación a la familia y los amigos, en definitiva, con una vida de relación normal fuera del trabajo. Sólo los que no se dejan ofuscar por "el engaño" del éxito a corto plazo $\mathrm{y}$, a base de tiempo y esfuerzo han llegado a disponer de un grado suficiente de autodominio, son capaces de encarar proyectos a largo plazo de envergadura y dignos. Junto a todo lo anterior, parece una necesidad que los estudiantes de medicina y los médicos especialistas en formación, adquieran una mayor madurez para comprender el significado que la salud y la enfermedad tiene para cada paciente, aprendiendo a considerar sus preferencias y a respetar sus valores. Sería interesante dar mayor peso curricular al cuidado del enfermo crónico y en situación terminal. Se debe insistir en el derecho de los pacientes a la información y el consentimiento informado, de acuerdo con las normas de cada país, dada la diversidad de legislación de los países de la UE. Deben ser tenidos en cuenta los principios éticos de la colaboración entre la profesión médica y la industria farmacéutica. Debe recordarse la obligación de los médicos de atenerse a las convenciones internacionales sobre los derechos humanos, recordando que los médicos prestarán la adecuada asistencia sanitaria, respetando el consentimiento y la confidencialidad de las personas que atiendan. También parece necesario potenciar algunas de las cualidades humanas del buen médico, como la compasión, la cortesía y la amabilidad. Además, la responsabilidad social de las instituciones sanitarias y universitarias exige también una gran capacidad de adaptación a las cambiantes necesidades de la comunidad, que no deben olvidarse. Con todo, conviene también tener en cuenta que la aplicación automática de un principio o la respuesta prefijada a un "caso-tipo" no resultan satisfactorias para afrontar los problemas éticos y bioéticos, entre otras razones, porque la realidad de la persona humana es mucho más rica que su formulación racional.

Los nuevos planes de estudios que se están diseñando para las Universidades requieren una reflexión conjunta de profesores, alumnos e Instituciones Sanitarias para que los valores del médico sean tenidos en cuenta al definir las competencias de salida. También lo requiere la confección de los programas de formación de especialistas para el período de la Residencia, considerándose por parte de las Comunidades Autónomas el diseño de formación horizontal complementaria si esto no se incluye por parte de las Comisiones nacionales de las especialidades.

\section{BIBLIOGRAFÍA}

1. Platón. Las leyes. Platón, obras completas. Madrid: Aguilar SA de Ediciones, 1969:1274-1520.

2. Miguel Angel Martí García. La Madurez. Dar a las cosas la importancia que tienen. Ediciones Internacionales Universitarias SA. Madrid. 1998.

3. Medical Professionalism Project, ABIM Foundation, ACPASIM Foundation, and European federation of Internal Medicine.. Ann Int Med 2002;136:243-246

4. Dyrbye LN, Thomas MR, Huntington JL, Lawson KL, Novotny PJ, Sloan JA, Shanafelt TD. Personal life events and medical student burnout: A multicenter study. Academic Medicine 2006;81:374-384

5. Swick HM, Bryan C S, Longo LD. Beyond the physician charter: reflections on medical professionalism. Perspectives in Biology an Medicine 2006;49:263-275.

6. Alexander L. Medical science under dictatorship. N Engl J Med 1949;241:39-47. 